Letter to the Editor

\title{
Use of a novel contact lens to improve fundal optical coherence tomographic images in keratoconus
}

Elena Y Zhu OD, ${ }^{1}$ Alexandros I oannidis FRANZCO, ${ }^{1}$ Peter Harrington BSc $\mathrm{RC}^{3}$ and Andrew Symons PhD FRANZCO ${ }^{1,2}$

1. Department of Ophthalmology, Royal Melbourne Hospital, Parkville, VIC, Australia

2. Department of Surgery, University of Melbourne, Australia

3. Ocular Instruments Inc., Bellevue, Washington, USA

Correspondence: Dr. Andrew Symons, Department of Ophthalmology, Royal Melbourne Hospital, 300 Grattan St, Parkville, VIC, 3050, Australia

Email: AndrewSymons@gmail.com

Received 13 February 2017; accepted 20 March 2017

Conflict of interest: A/Prof Symons declares that his eponymous lens was developed from his original concept and that Peter Harrington of Ocular Instruments Inc. contributed to the design. A/Prof Symons does not have a financial relationship with Ocular and does not have a financial interest in this lens. Peter Harrington is an employee of Ocular.

Funding sources: None

This is the author manuscript accepted for publication and has undergone full peer review but has not been through the copyediting, typesetting, pagination and proofreading process, which may lead to differences between this version and the Version of Record. Please cite this article as doi: 10.1111/ceo.12949

This article is protected by copyright. All rights reserved. 
The use of a novel contact lens to aid optical coherence tomographic imaging of the ocular fundus in keratoconus patients is described in this case report. It is anticipated that this lens will be beneficial for imaging in other tectonic corneal disorders by reducing the aberration caused by irregular astigmatism.

A 38-year-old Caucasian female presented for evaluation of reduced vision in the left eye. At the age of five she had a low-grade astrocytoma of the optic chiasm debulked, with the exception of unresectable tumour of the left optic nerve. She was diagnosed with keratoconus at 29 and refused penetrating keratoplasty.

On examination, her best corrected visual acuities were $6 / 12$ with $+1.00 /-3.00 \times 75^{\circ}$ in the right eye and $6 / 15$ with $+1.75 /-7.5 \times 110^{\circ}$ in the left eye. With pinhole, visual acuities reached 6/9 bilaterally. Automated perimetry, Placido disc based corneal topography (Orbscan, Bausch\& Lomb, NY, USA) and optical coherence tomography (OCT) (Spectralis, Heidelberg Engineering, Heidelberg, Germany) of the maculae and peripapillary retinal nerve fibre layers were ordered. Automated perimetry demonstrated a possible right homonymous hemianopia. Corneal topography (Figure 1) demonstrated bilateral large paracentral cones, consistent with the diagnosis of keratoconus.

Image degradation rendered OCT images of little diagnostic benefit. Therefore, OCT were repeated with a single element quartz $(n=1.46)$ ocular contact lens (Symons I mage Enhancing Lens, product code: OSIE, Ocular Instruments Inc., Bellevue, WA, USA) designed to negate aberrations due to the keratoconic cornea. The lens has a central thickness of $1.0 \mathrm{~mm}$, a net dioptric power of zero and incorporates a $20 \mathrm{~mm}$ 
diameter flange to facilitate clinical control of eye movement. The anterior surface of the lens has a high performance anti-reflection coating that minimizes reflection intensity at a wavelength of $830 \mathrm{~nm}$. The patient's eyes were anaesthetized with topical oxybuprocaine and Hypromellose $0.3 \%$ (Genteal gel, Novartis, Basel, Switzerland) was used as an optical medium between the cornea and the posterior surface of the lens. High quality OCT images were captured with this lens (Figure 2), demonstrating normal macular anatomy and borderline retinal nerve fiber layer.

It was concluded that the progressive visual loss was related to worsening keratoconus rather than progressive optic neuropathy.

Visual function may be reduced by optical consequences of tectonic corneal dystrophies such as keratoconus. In the case described, the optical imperfections of the keratoconic corneas also impaired the OCT signal to levels of diagnostic futility. Previous studies demonstrate that refractive errors may alter OCT signal strength. Hwang and coworkers have demonstrated that contact lens induced astigmatism reduces OCT signal strength, ${ }^{1}$ and Uzunel et al. ${ }^{2}$ also shown that keratoconus may reduce OCT signal strength and image quality. Although a subset of keratoconic patients studied by Sahebjada et al..$^{5}$ wore their refractive contact lenses while OCT scans were performed, previous studies have not determined the impact of diagnostic fundal contact lens use on OCT measurement quality in patients with keratoconus.

Our case demonstrated that neutralization of the anterior irregularity of a severely keratoconic cornea with a fundal contact lens was sufficient to overcome the keratoconus induced degradation of the OCT signal.

Interestingly, studies comparing OCT measurements in normal and keratoconus patients yielded inconsistent results, with keratoconus patients having either reduced ${ }^{2}$ or increased ${ }^{4}$ macular thickness compared to controls. The degree to which these results were influenced by the use or lack thereof of patients' regular contact lenses 
during OCT scanning is unknown. Further investigation using a fundal contact lens neutralising the effect of the anterior corneal curvature may aid in determining the degree to which keratoconus is associated with altered macular anatomy.

To our knowledge, this is the first report of a specially designed contact lens aiding OCT imaging of the ocular fundus. Potential users of this lens are recommended to have a second eye-care professional available to stabilize the fundal contact lens during use. The lens of the OCT instrument is expected to come within a few millimeters of the flange of the contact lens.

\section{REFERENCES}

1. Hwang YH, Lee SM, Kim YY, Lee JY, Yoo C. Astigmatism and optical coherence tomography measurements. Clinical Experimental Ophthalmology. 2012 Feb;250(2):247-54.

2. Uzunel UD, Küsbeci T, Yüksel B. Does the Stage of Keratoconus Affect Optical Coherence Tomography Measurements? Semin Ophthalmol. 2016 J ul 1:1-6.

3. Salchow DJ , Li FY, Hwang AM, Dziura J. Effect of contact lens power on optic disc parameters measured with optical coherence tomography. Current Eye Research, 2013 Mar; 38(3):381-5.

4. Lee J, Kim NR, Kim H, Han J, Lee ES, Seong GJ, Kim CY. Negative refraction

- power causes underestimation of peripapillary retinal nerve fibre layer thickness in spectral-domain optical coherence tomography. British J ournal of Ophthalmology, 2011 Sep; 95(9):1284-9 
5. Sahebjada S, Amirul Islam FM, Wickremasinghe S, Daniell M, Baird PN.

Assessment of Macular Parameter Changes in Patients with Keratoconus Using Optical Coherence Tomography. J Ophthalmol. 2015

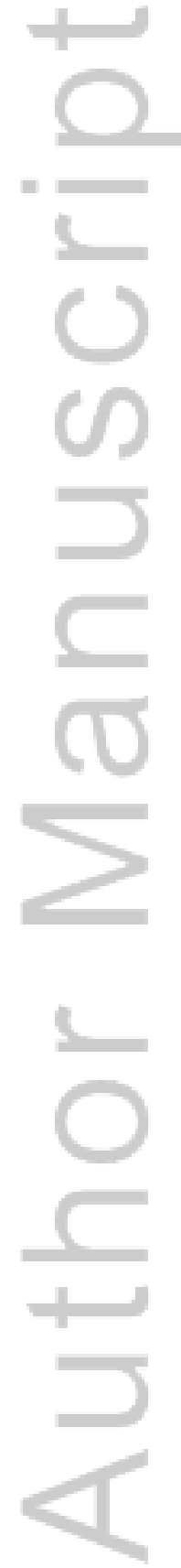

This article is protected by copyright. All rights reserved. 


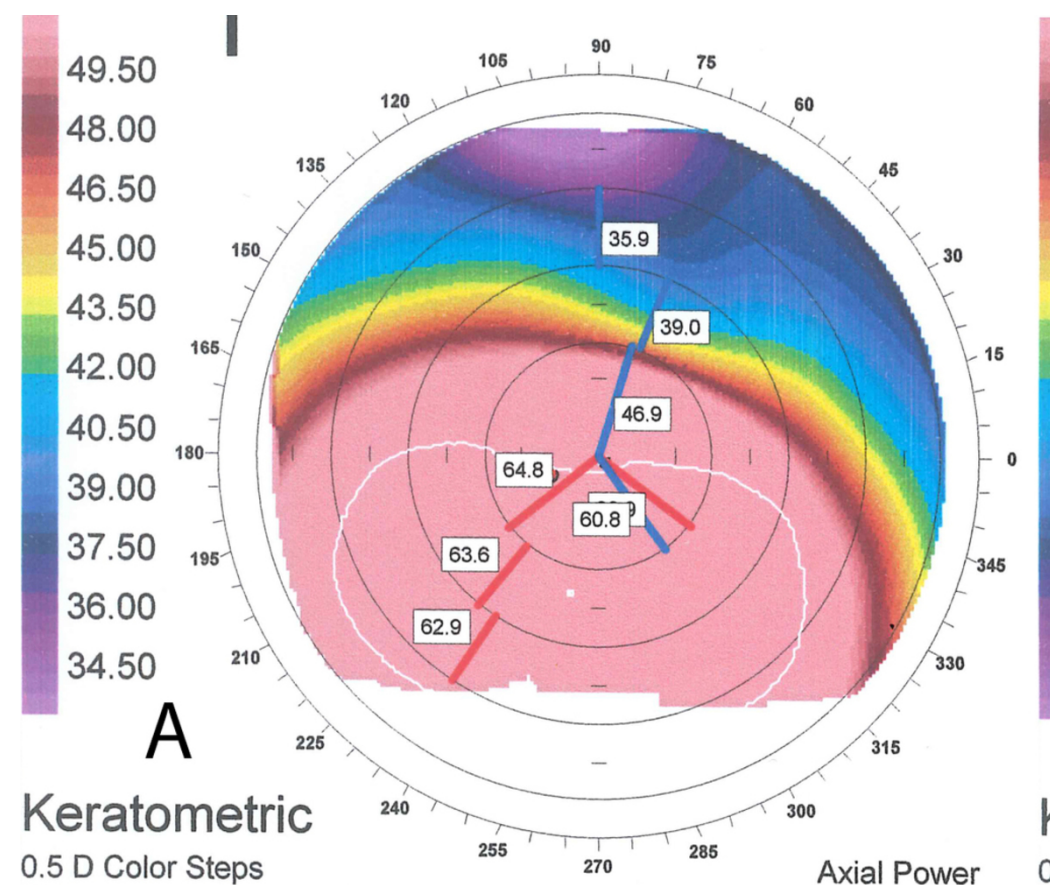

$0.5 \mathrm{D}$ Color Steps

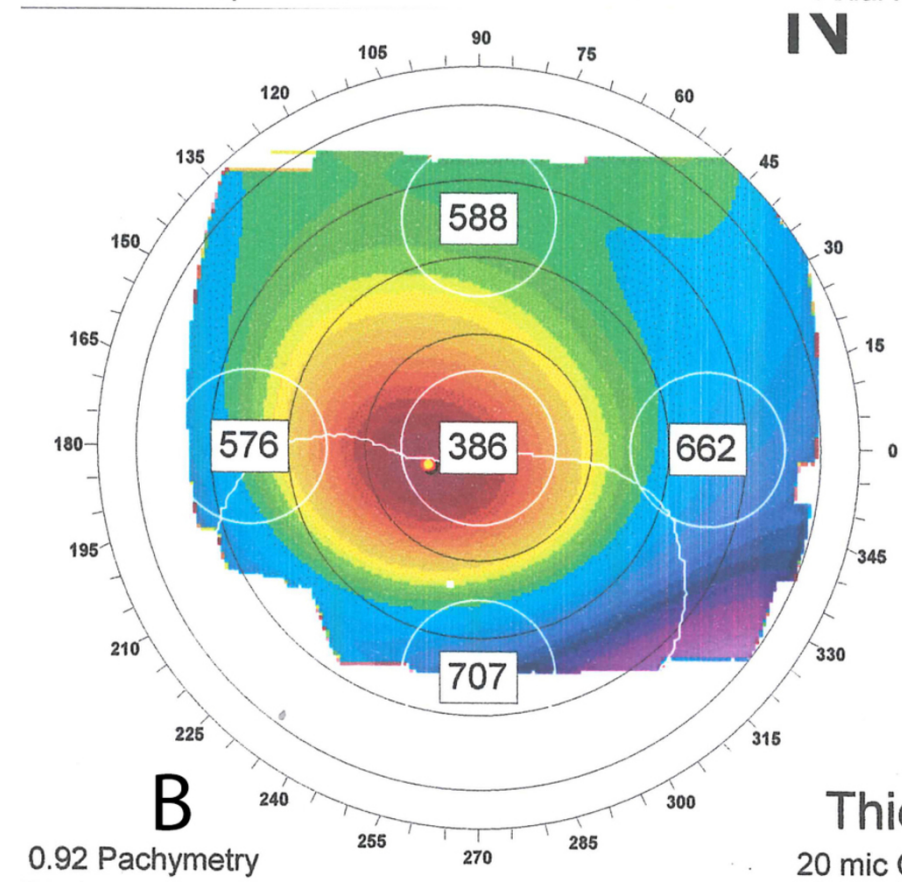

Thickness

20 mic Color Steps .92 Pachymetry

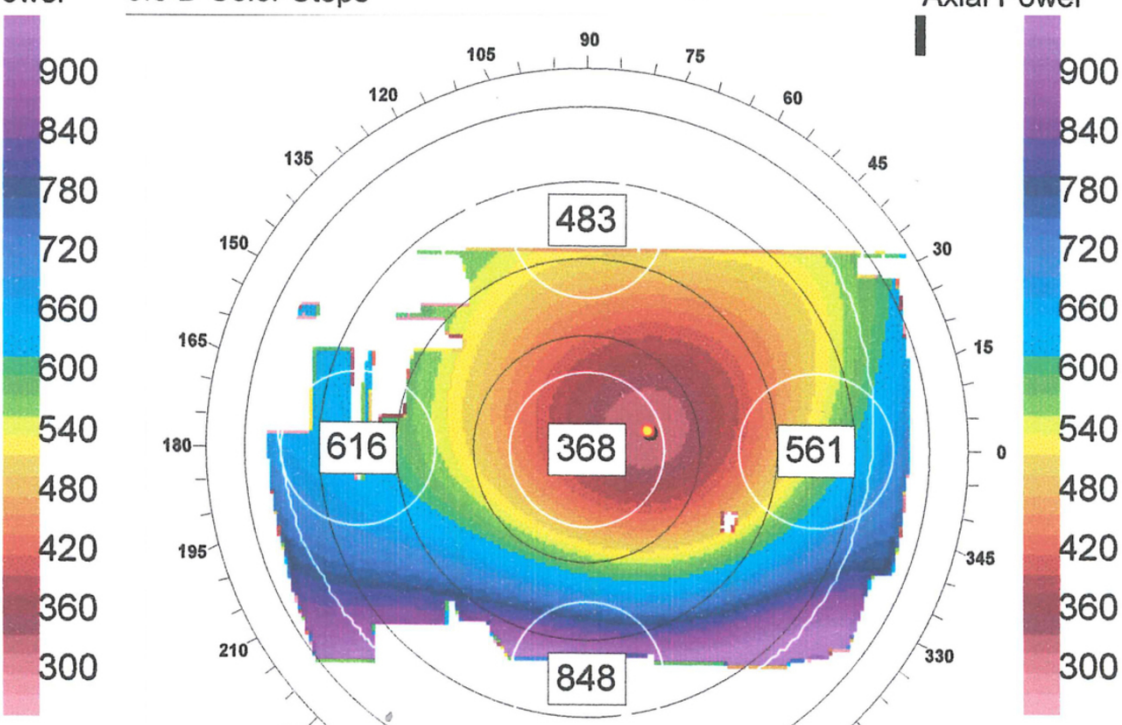

CE0_12949_F1.jpg
IV

48.00

46.50

45.00

43.50

42.00

40.50

39.00

37.50

36.00

34.50

C

Keratometric

225
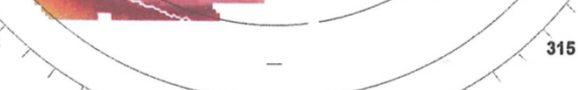
315

300

Axial Power 
A
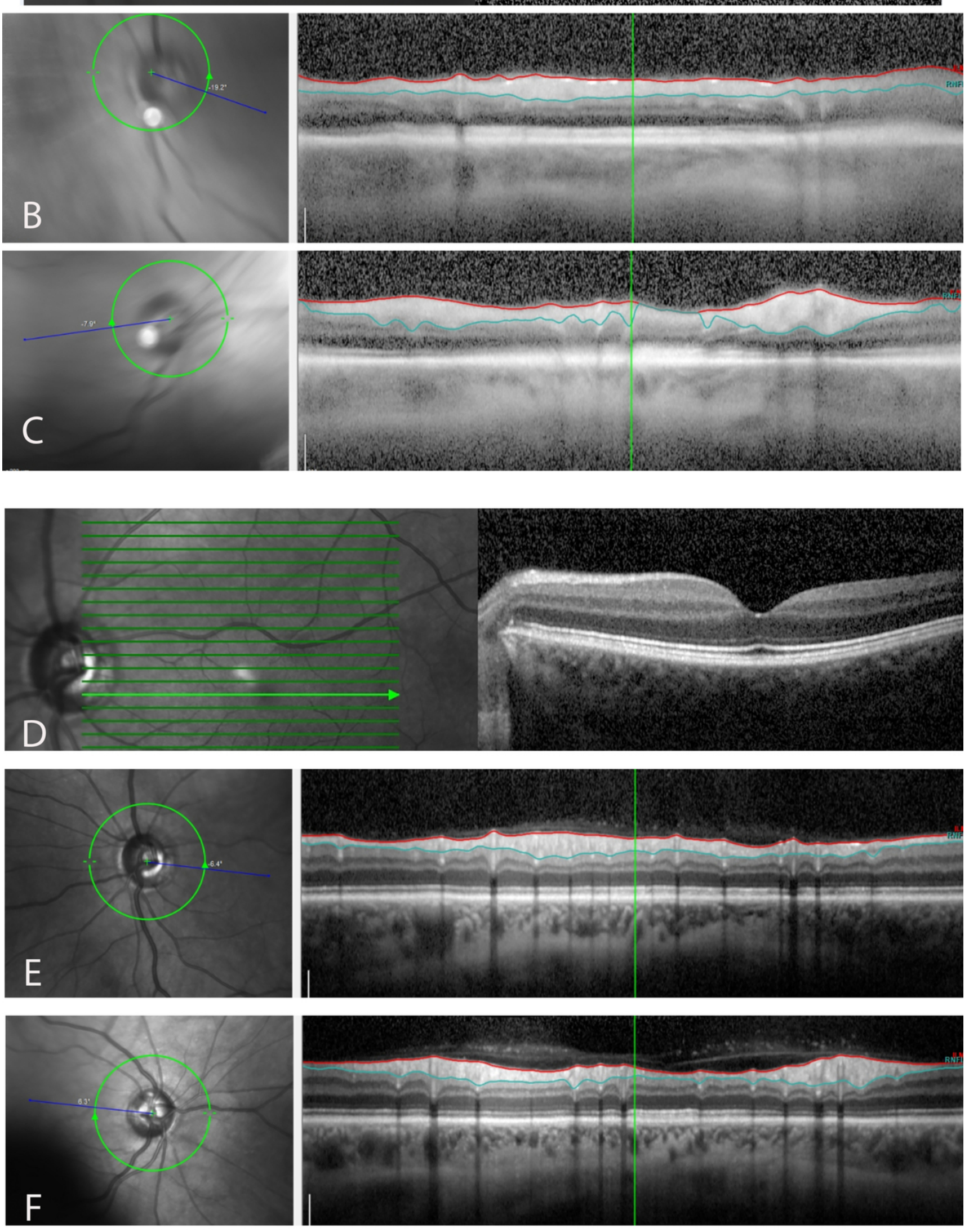

CEO_12949_F2.jpg

This article is protected by copyright. All rights reserved. 

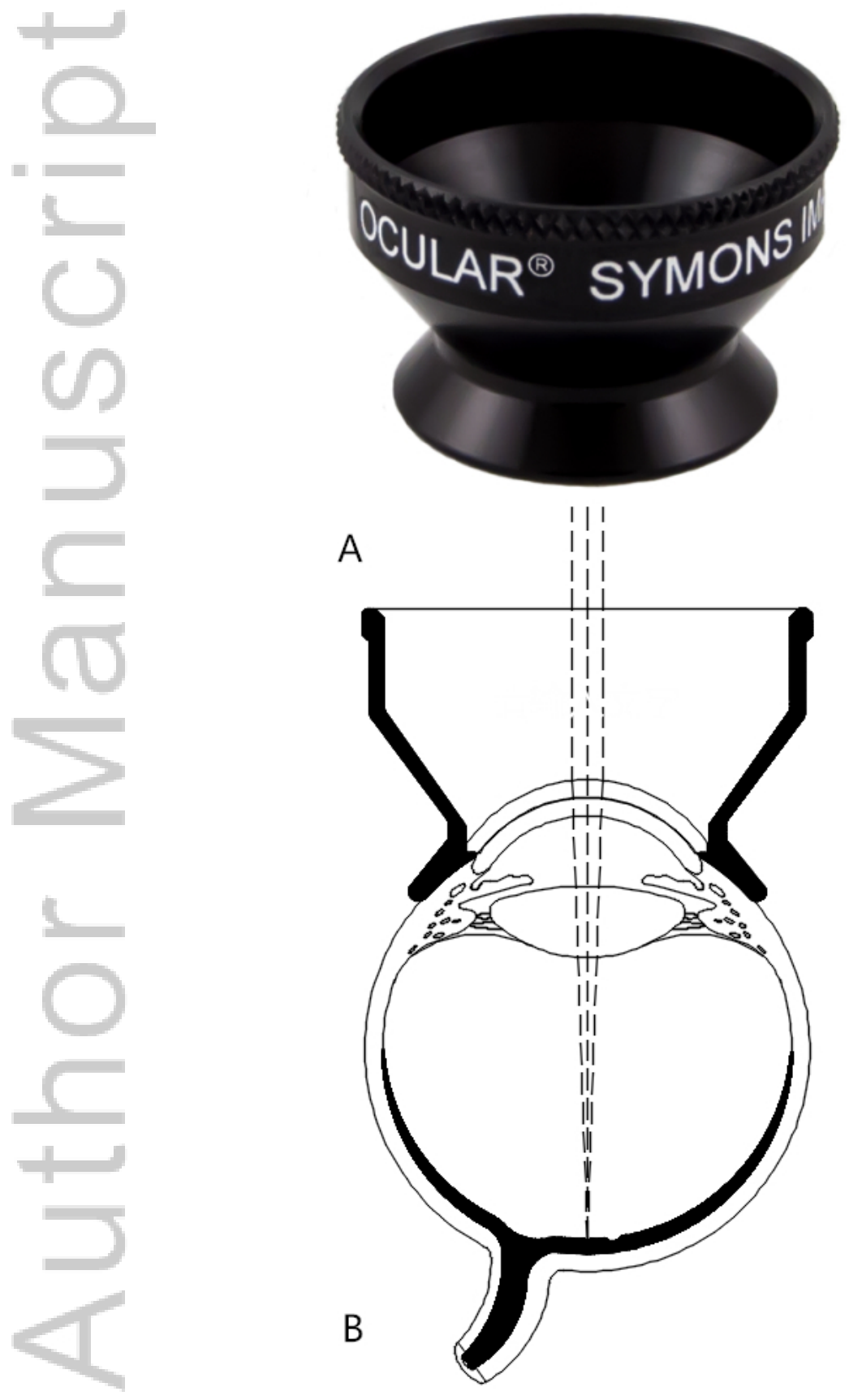

CE0_12949_F3.jpg

This article is protected by copyright. All rights reserved. 


\section{University Library}

\section{- M M N E R VA A gateway to Melbourne's research publications}

Minerva Access is the Institutional Repository of The University of Melbourne

Author/s:

Zhu, EY;loannidis, A;Harrington, P;Symons, RCA

Title:

Use of a novel contact lens to improve fundal optical coherence tomographic images in keratoconus

Date:

2017-09-01

Citation:

Zhu, E. Y., loannidis, A., Harrington, P. \& Symons, R. C. A. (2017). Use of a novel contact lens to improve fundal optical coherence tomographic images in keratoconus. CLINICAL AND EXPERIMENTAL OPHTHALMOLOGY, 45 (7), pp.742-745. https://doi.org/10.1111/ ceo.12949.

Persistent Link:

http://hdl.handle.net/11343/292883 